\section{Scurvy as the Presenting Illness of Whipple's Disease Exacerbated by Treatment with Etanercept in a Patient with Ankylosing Spondylitis}

To the Editor:

A 35-year-old man was diagnosed with ankylosing spondylitis (AS) in 2004. HLA-B27 typing was negative. He started therapy with etanercept $25 \mathrm{mg}$ twice weekly subcutaneously in September 2008 because of highly active disease. Two months after starting etanercept, the condition was not improved. He was hospitalized because of sudden onset of a gingival nodule (Figure 1A), and purpura (Figure 1B), and a history of abdominal pain with 3 non-bloody stools per day, fatigue, early satiety, and a $6 \mathrm{~kg}$ weight loss during the preceding 4 months. Etanercept was then stopped.

On examination at our institution, his temperature was $37.5^{\circ} \mathrm{C}$, blood pressure $120 / 80 \mathrm{~mm} \mathrm{Hg}$, pulse 88 beats per minute, and weight $66 \mathrm{~kg}$. The spleen edge was palpable $2 \mathrm{~cm}$ below the costovertebral margin. He had lower limb purpura, lower limb edema, and gingivitis. Results of abdominal and neurological examination were normal. Laboratory studies showed erythrocyte sedimentation rate (ESR) $82 \mathrm{~mm} / \mathrm{h}$ (Westergren), C-reactive protein (CRP) $78.9 \mathrm{mg} / \mathrm{l}$ (normal 0-6 mg/l), hemoglobin $9.3 \mathrm{~g} / \mathrm{dl}(13.5-17.5$ $\mathrm{g} / \mathrm{dl}$ ). Vitamin C was $5 \mu \mathrm{mol} / 1$ (reference range $55-72 \mu \mathrm{mol} / 1$ ), with hypoalbuminemia (27 g/l) and hypoprotidemia (53 g/l) secondary to malabsorption. Immune tests were negative. Radiograph of the pelvis showed grade 2 sacroiliitis. Computed tomography scan of the abdomen revealed multiple enlarged retroperitoneal, mesenteric, and celiac nodes (Figure 2A). There was no abnormality on the brain on magnetic resonance imaging.

Acute pancolitis was observed on colonoscopy, and histological evaluation revealed macrophages that stained positive with periodic acid-Schiff with diastase digestion (PAS-D). Polymerase chain reaction (PCR) testing for Tropheryma whipplei was positive in blood, stool, and cerebrospinal fluid.

The patient reported a 15-year history of skin lesions on his right elbows. Large biopsy of this nodule showed granulomatous nodules admixed with lymphocytes in the deep dermis with septal panniculitis. This noncaseating granulomatous inflammation was first diagnosed as sar- coidosis. Secondarily we observed that these cells were mostly CD68-positive and contained PAS-positive intracytoplasmic material compatible with Whipple's disease.

Based on the clinical presentation and the endoscopic and histological findings, a diagnosis of Whipple's disease with scurvy involvement was made.

Upon treatment with trimethoprim $(160 \mathrm{mg})$ and sulfamethoxazole $(800 \mathrm{mg}$ ) orally twice daily, the systemic symptoms and polyarthritis progressively resolved over 1 month and CRP and ESR normalized. Skin lesions disappeared rapidly within 10 days (Figure 1D). Three months after starting antibiotics, arthralgia, diarrhea, weight loss, and fever were completely resolved with less lymphadenopathy in the peripancreatic and around the celiac axis (Figure 2B ).

Whipple's disease is a rare infectious disease caused by the bacillus $T$. whipplei. Only a few studies have examined consecutive patients with Whipple's disease for sacroiliitis and $\mathrm{AS}^{1,2}$. To exclude definitively the association of Whipple's disease with AS it is necessary to obtain bacteriological data on T. whipplei before the diagnosis of AS. Involvement of scurvy (by vitamin $\mathrm{C}$ deficiency), which is only occasionally described, typically occurs late in the disease course ${ }^{3}$. Hemorrhagic syndrome and edema were described in patients with vitamin $\mathrm{C}$ deficiency. Cutaneous biopsies have typical aspects of scurvy in histopathology. If a patient has received immunosuppressive therapy, such as treatment with corticosteroids or tumor necrosis factor (TNF) antagonists, a more rapid clinical progression may occur ${ }^{4}$. In our case, systemic manifestations of scurvy that appeared following initiation of etanercept therapy suggest an exacerbation of uncontrolled T. whipplei infection. Discontinuation of the anti-TNF drug associated with introduction of antibiotics allowed control of the infection.

To date, few cases of Whipple's disease after initiation of anti-TNF therapy have been described ${ }^{5-8}$. This has confirmed the effect of anti-TNF drugs on worsening infections such as those with intracellular organisms or the granulomatous ones.

Our patient had had a subcutaneous nodule 15 years earlier and electron microscopy and PCR had confirmed the presence of T. whipplei. T. whipplei has been characterized by amplification of its unique 1321-base

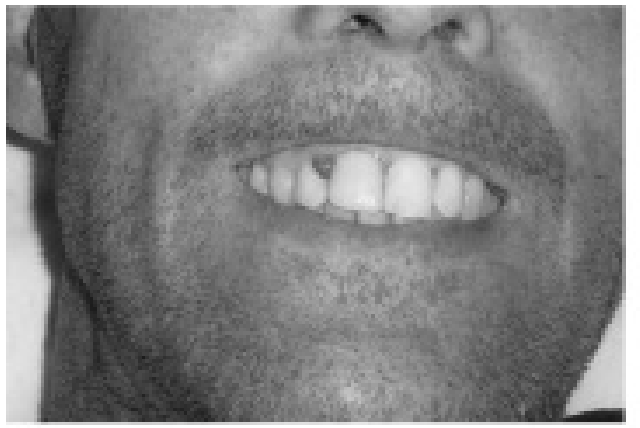

A

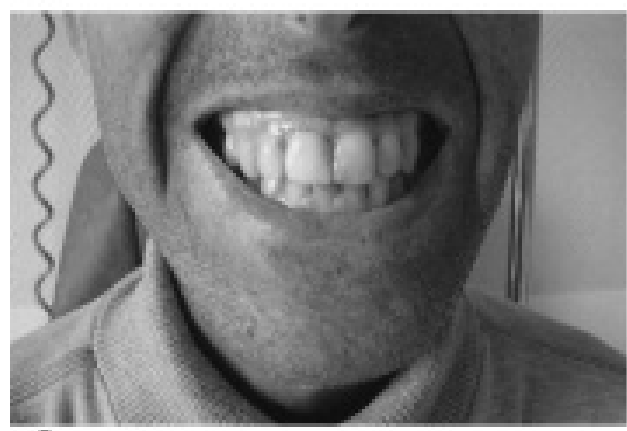

C

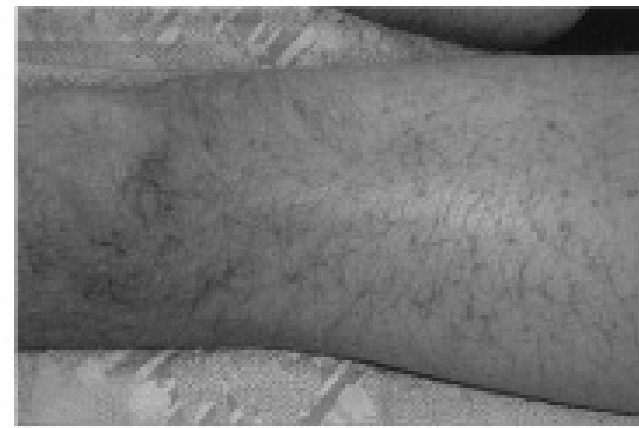

B

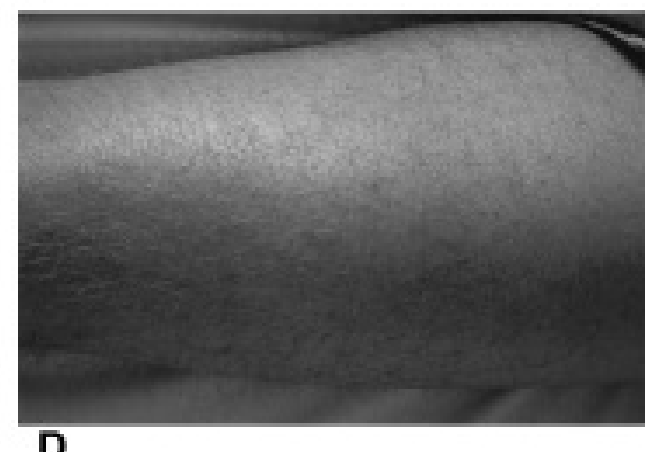

Figure 1. Clinical cutaneous manifestations associated with vitamin C deficiency before treatment (A, B) and reduction of these manifestations after 10 days of treatment $(C, D)$. 

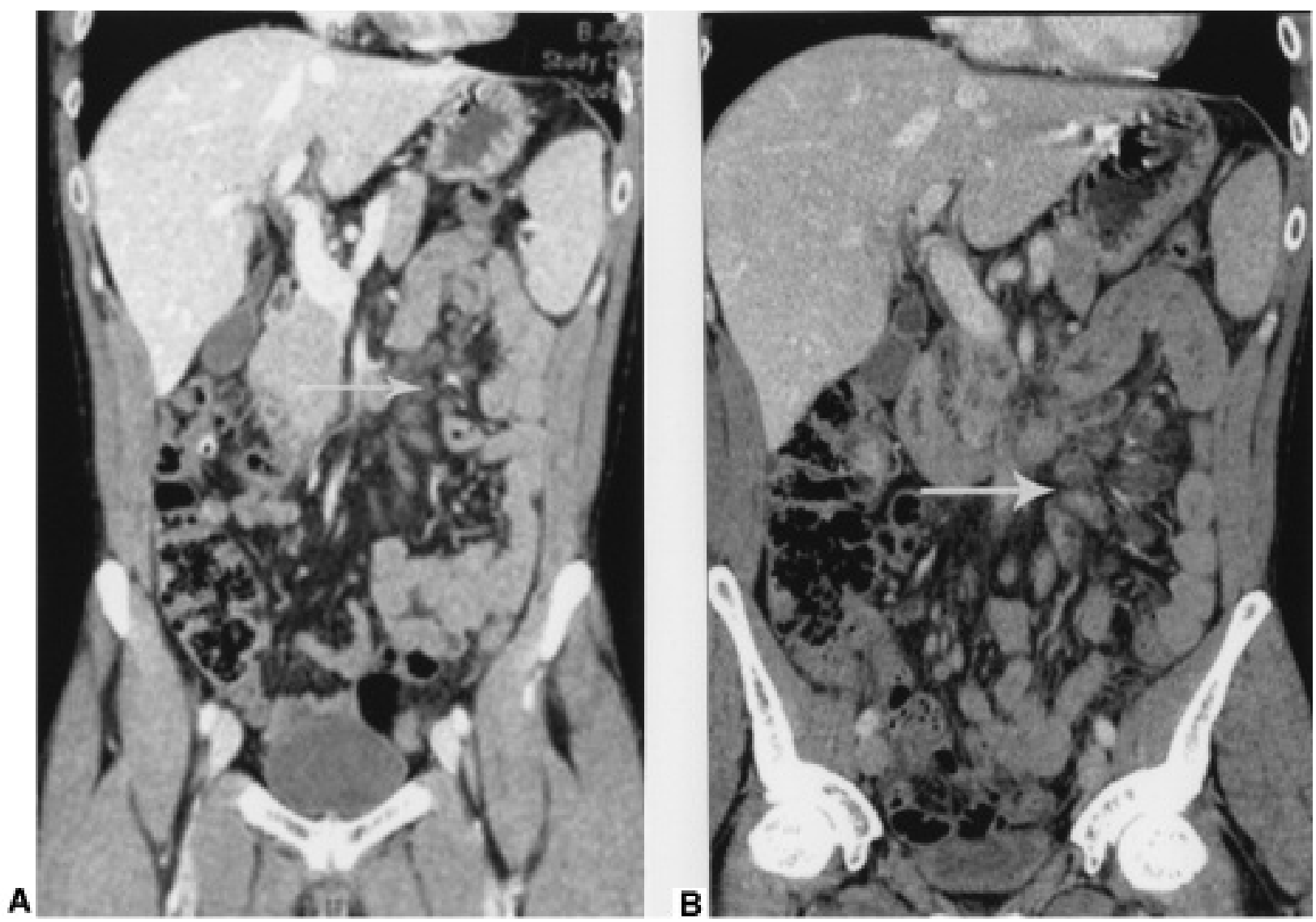

Figure 2. CT scans of the abdomen with contrast enhancement show lymphadenopathy around the celiac axis (arrow, A). Image obtained after treatment shows reduction in size of the lymphomas (arrow, B).

sequence of the 16S rRNA (rRNA, small subunit) gene from infected tissue $^{9}$. This presents an alternative for diagnosing Whipple's disease in cases with atypical clinical features or when the histological diagnosis requires confirmation. Complete sequencing of the T. whipplei genome has allowed development of PCR based on more specific genes and increased sensitivity with the use of repeated sequences ${ }^{9}$. Such subcutaneous involvement by Whipple's disease is very rare ( 4 documented cases) and can be misdiagnosed as sarcoidosis ${ }^{10}$.

To our knowledge, we describe the first case of Whipple's disease in which manifestations of scurvy appeared after initiation of etanercept therapy in a patient with AS. Moreover, this infection has been well documented with evidence of T. whipplei on actual digestive lesions and on a subcutaneous nodule 15 years before the diagnosis of AS was established. Etanercept probably modified the cytokine environment and thus favored the exacerbation of Whipple's disease.

IHSANE HMAMOUCHI, MD, Department of Immuno-Rheumatology, Lapeyronie Hospital; VALÉRIE COSTES, MD, PhD, Department of Hematology and Pathology, INSERM 475 and CHU 34295; BERNARD COMBE, MD, $\mathrm{PhD}$; JACQUES MOREL, MD, $\mathrm{PhD}$, Department of Immuno-Rheumatology, Lapeyronie Hospital, Montpellier, France. Address correspondence to Dr. Hmamouchi; E-mail: i.hmamouchi@yahoo.fr

Supported by the Unité des Rickettsies, IFR 48, Centre National de la Recherche Scientifique UMR 6020, and Université de la Méditerranée, Marseille, France. We express thanks to Dr. Florance Fenollar for her contribution.

\section{REFERENCES}

1. d'Eshougues JR, Delcambre B, Defrance D. Articular manifestations of Whipple's disease. Rev Rhum Mal Osteoartic 1976;43:565-73.

2. Khan MA. Axial arthropathy in Whipple's disease. J Rheumatol 1982;9:928-9.

3. Berger ML, Siegel DM, Lee EL. Scurvy as an initial manifestation of Whipple's disease. Ann Intern Med 1984;100:58-9.

4. Mahnel R, Kalt A, Ring S, Stallmach A, Strober W, Marth T. Immunosuppressive therapy in Whipple's disease patients is associated with the appearance of gastrointestinal manifestations. Am J Gastroenterol 2005;100:1167-73.

5. Kneitz C, Suerbaum S, Beer M, Müller J, Jahns R, Tony HP. Exacerbation of Whipple's disease associated with infliximab treatment. Scand J Rheumatol 2005;34:148-51.

6. Legoupila N, Jourdana C, Poyart C, Ariche L, Puéchal X, Job-Deslandre X, et al. Atteinte cutanée au cours de la Maladie de Whipple: une manifestation exceptionnelle. Rev Rhum Mal Osteoartic 2006;73:1232.

7. Kremer AE, Budenhofer U, Beuers U, Rust C. A 47-year-old dog breeder with chronic polyarthritis, weight loss and high fever. Z Gastroenterol 2008;46:431-4.

8. Ahmadi-Simab K, Schnitzler P. Whipple's disease with normal duodenal histology and AS. Dtsch Med Wochenschr 2009; 134:127-30.

9. Fenollar F, Fournier PE, Raoult D, Gerolami R, Lepidi H, Poyart C. Quantitative detection of Tropheryma whipplei DNA by real-time PCR. J Clin Microbiol 2002;40:1119-20.

10. Tarroch X, Vives P, Salas A, Moré J. Subcutaneous nodules in Whipple's disease. J Cutan Pathol 2001;28:368-70.

J Rheumatol 2010;37:5; doi:10.3899/jrheum.091301 